\title{
Ecoeficiencia de la infraestructura hidráulica del sistema Chancay-Lambayeque y su impacto en la huella hídrica de la producción agrícola
}

\author{
Eco-efficiency of the hydraulic infrastructure of the Chancay- \\ Lambayeque system and its impact on the water footprint of \\ agricultural production
}

Recibido: setiembre 03 de 2020 | Revisado: setiembre 28 de 2020 | Aceptado: octubre 02 de 2020

Juan Delgado $^{1}$
Walter Campos $^{2}$
Alexis Dueñas $^{3}$

1 Pontificia Universidad Católica del Perú

2 U n i v e r i d a d Nacional Pedro Ruiz Gallo. Departamento de Educación

3 Universidad Nacional

Agraria La Molina, Departamento de Horticultura fduenas@lamolina.edu. pe

\section{RESUMEN}

Los recursos hídricos se ven afectados por las variaciones climáticas, en particular por la magnitud y distribución de precipitaciones, aumento de temperatura y mayor probabilidad de ocurrencia de eventos extremos. La huella hídrica es un indicador que expresa el uso de agua tanto en su uso directo, como indirecto y para su cálculo se empleó el software CROPWAT 8.0 y la base de datos CLIMWAT 2.0. Se estimó que la huella hídrica total de los cultivos estudiados en $\mathrm{m}^{3}$.ton ${ }^{-1}$ de producto generado es de 156,1124 y 891 para la cańa de azúcar, el arroz y el maíz respectivamente; con una contribución de la huella verde en $1.6 \%, 0.7 \%$ y $3.4 \%$. El cultivo con mayor huella es el arroz y la caña de azúcar tiene la menor huella hídrica, explicado por altos rendimiento en biomasa. Con base en la cantidad de agua requerida por superficie cultivada, se aprecia que la caña de azúcar es el cultivo con mayor consumo, al requerir $16000 \mathrm{~m}^{3}$.ha-1 arroz y el maíz solo requieren 9000 y $6000 \mathrm{~m}^{3}$.ha-1 de agua respectivamente. Se determinó que la eficiencia del arroz es $3.24 \mathrm{~m}^{3} \cdot$ ton $^{-1}$ de agua por volumen producido. La comisión de Morrope presenta una mejor relación entre volumen de producción y volumen de agua, con ratios de 2.57 y 2.52 en comparación con el resto, que tienen valores de 1.25 y 1.21 para el arroz y el maíz, respectivamente.

Palabras clave: Recursos hídricos, huella hídrica, ecoeficiencia

\begin{abstract}
Water resources are affected by climate variations, in particular by the magnitude and distribution of precipitation, increased temperature and likelihood of occurrence to extreme events. The water footprint is an indicator that expresses direct and indirect water use. In addition cropWAT 8.0 software and CLIMWAT 2.0 database were used for its calculation. The total water footprint of crops studied in $\mathrm{m}^{3} \cdot$ ton $^{-1}$ of the product generated was estimated to be 156,1 124 and 891 for sugarcane, rice and maize respectively; with a green footprint contribution of $1.6 \%, 0.7 \%$ and $3.4 \%$. The largest crop is rice and sugar cane has the lowest water footprint, explained by high biomass yields. Based on the amount of water required per cultivated area, sugar cane is found to be the most consumed crop, requiring $16,000 \mathrm{~m}^{3} \cdot \mathrm{ha}^{-1}$, rice and maize require only 9,000 and $6,000 \mathrm{~m}^{3} \cdot \mathrm{ha}^{-1}$ of water respectively. Rice efficiency was determined to be 3.24 $\mathrm{m}^{3} \cdot \operatorname{ton}^{-1}$ of water per volume produced. Morrope's commission has a better ratio of production volume to water volume, with ratios of 2.57 to 2.52 compared to the rest, which have values of 1.25 and 1.21 for rice and maize, respectively.
\end{abstract}

Key words: Water resources, water footprint, eco-efficiency

(C) Los autores. Este artículo es publicado por la Revista Campus de la Facultad de Ingeniería y Arquitectura de la Universidad de San Martín de Porres. Este artículo se distribuye en los términos de la Licencia Creative Commons Atribución No-comercial - Compartir-Igual 4.0 Internacional (https://creativecommons.org/licenses/ CC-BY), que permite el uso no comercial, distribución y reproducción en cualquier medio siempre que la obra original sea debidamente citada. Para uso comercial contactar a: revistacampus@usmp.pe. 


\section{INTRODUCCIÓN}

La UNESCO define a los recursos hídricos como aquellos relativos al agua, disponibles o potencialmentedisponibles, en cantidad y calidad suficiente, en un lugar y periodo de tiempos apropiados para satisfacer una demanda identificable (NNUU, 2017). El uso adecuado de los recursos naturales es la base de las dimensiones del desarrollo sustentable, tales como la gobernabilidad, salud humana, conservación de la biodiversidad y adaptación al cambio climático (FAO, 2018). Para el caso de la agricultura, los más relevantes son el agua y el suelo, de modo que, el agua es el insumo de mayor volumen y consumo en la producción de alimento, tanto en el campo como en la mayoría de los clústeres de la cadena de valor.

Los recursos naturales deben preservarsey utilizarse de manera racional, al ser imprescindibles para el desarrollo de la vida y la realización de actividades humanas (Effendi, 2016). El Informe de las Naciones Unidas sobre el Desarrollo de los Recursos Hídricos en el Mundo, señala que del total de agua existente en el planeta, el $97.5 \%$ se encuentra en los mares y océanos, se trata básicamente de agua salada y el agua dulce representa el 2.5\%; de esta, el $68.7 \%$ se encuentra en los glaciares de los casquetes polares y las cumbres nevadas, el $30.1 \%$ está en acuíferos subterráneos, un $0.8 \%$ en el permafrost y el $0.4 \%$ restante en aguas superficiales y en la atmósfera (NNUU, 2017).

Un problema adicional, que afecta el uso sostenible del agua, es la disposición física agua en el planeta, que alude a sus patrones de distribución. Alrededor de 600 millones de personas carecen de un acceso seguro al agua para consumo doméstico por el limitado tratamiento (UNICEF, 2018.). Esto se debe, en parte, a la distribución irregular de las precipitaciones, tanto espacial como temporalmente (McMichael, 2014); pero también a la carencia de sistemas de infraestructura de abastecimiento y mecanismos de gestión que permitan el acceso al recurso a poblaciones vulnerables (Massoud et al. 2010). A ello se añade, el problema de la presión hídrica, indicador que mide en términos del porcentaje el agua extraída respecto a la disponibilidad natural media total (Bunge, 2010).

La contaminación frecuente en la agricultura resultante del uso inadecuado de plaguicidas en las prácticas agrícolas es la principal fuente de contaminación no puntual del agua subterránea por lixiviación (Bedmar et al.2015). También, se ha determinado que el uso de suelos y al regadío vinculada al cultivo contribuyen significativamente con la elevada salinidad de los flujos de agua (Larsen et al. 2013). Otro aspecto se relaciona con la sobreexplotación de fuentes hídricas, tanto superficial como subterránea, que conllevan al agotamiento del recurso, como es el caso de la sobreexplotación de aguas subterráneas (Gleeson et al. 2016).

A los problemas descritos se incorpora los efectos derivados del cambio climático, en particular para el caso de la agricultura, actividad que depende en extremo de las condiciones ambientales. Así se tiene, que las variaciones climáticas, observadas en el último medio siglo, de la magnitud y distribución de precipitaciones, aumento de la temperatura y mayor probabilidad de eventos extremos como inundaciones, sequías, olas de frío y calor, entre otros 
(Pachauri et al. 2014), eventos que explican las alteraciones en la distribución de especies vegetales y perturbaciones en la fenología de diversos cultivos, limitando su crecimiento y sus rendimientos (Altieri et al. 2009). Por tanto, asegurar su disponibilidad es una prioridad global (Nieto, 2011) y estrategias como la captación de agua de lluvia (RojasValencia et al. 2012); recarga de acuíferos (Ponce et al. 2018); implementación de infraestructura (represas y embalses) para el almacenamiento y la generación de energía y control de inundaciones (Bedoya et al. 2015) también son alternativas útiles para mitigar la escasez de agua, como es el caso de la costa hiperárida del Perú (Álvarez et al. 2014), al incidir positivamente en el balance hidrológico. Además, no debe soslayarse, la reutilización de aguas servidas para el riego y la refrigeración industrial, favoreciendo el aseguramiento de la sostenibilidad ambiental (WHO, 2006) y en particular, la desalinización del agua de mar, con los avances tecnológicos que permiten abaratar los costos (Subramani et al. 2015).

A pesar de lo dicho, el diseño de una estrategia de gestión del recurso hídrico implica la medición de las extracciones, en ese sentido, la huella de agua es un concepto de importancia. El Foro Económico Mundial indica, en su décimo primer Reporte de Riesgos Globales, que la crisis del agua se encuentra entre los diez riesgos más relevantes para la humanidad (World Economic Forum, 2016). Además, se ha evidenciado que la irrigación es la actividad antrópica que conlleva a consecuencias ambientales importantes, al ser responsable del 85\% del consumo global y del $70 \%$ de la extracción (Bruinsma et al. 2012). En respuesta a este problema, el 2014 la
ISO publico la norma 14046, como una opción metodológica para evaluar los impactos ambientales potenciales de productos, servicios $\mathrm{u}$ organizaciones. En esta norma se define la huella de agua como el indicador que cuantifica los impactos ambientales potenciales relacionados con el uso y consumo del agua, considerando las etapas del ciclo de vida (ISO, 2014). La metodología descrita por la norma ISO 14046 se sustenta en un análisis integral de los impactos ambientales, relacionados al consumo del agua y depende de conceptos como la "huella ecológica" (Rees, 1992), el "agua virtual" (Allan, 1998), y la "huella hídrica” (Hoekstra, 2003).

Una de las metodologías, derivadas de la huella de agua, es la "huella de escasez de agua", que se encuentra en la base conceptual de los estudios de ACV o LCA (Bayart et al. 2010; Kounina et al. 2013) y se enfoca en el desarrollo de un indicador basado en la escasez, que sea calculable con base en los consumos de agua y que además, sea útil como punto de partida para diferentes tipos de análisis. En este contexto, la metodología propuesta por el grupo de trabajo del Uso del Agua en la Evaluación de Ciclo del Vida (WULCA), por sus siglas en inglés, incorpora la perspectiva del ACV, respecto a los impactos potenciales del consumo de agua de carácter interregional, dicho de otro modo, el impacto potencial de privar a un usuario de agua dulce, al consumir el recurso en otra región (Boulay et al. 2015). En esa línea argumentativa, se acuñan expresiones vinculadas a las extracciones (Frischknetch et al. 2009; Pfister et al. 2009), los consumos (Hoekstra et al. 2012; Berger et al. 2014) y la demanda de agua (Boulay et al. 2015). 
En tiempos recientes, se demostró que la mejor expresión para estimar la huella de agua debía basarse en el método Available WAter REmaining (AWARE), desarrollado por Boulay et al. (2018), que considera la disponibilidad menor de agua, por sus silgas en inglés "AMD" o availability minus demand. El agua disponible está expresada por la diferencia entre la precipitación efectiva y la evapotranspiración en cierto entorno geográfico, es decir, representa a todo volumen de agua aprovechable para el uso humano en un lugar determinado; en tanto que la demanda de agua se refiere al volumen necesario para satisfacer todas las necesidades de la comunidad o sociedad. Este indicador, según sus autores, cubre los vacíos reportado en la teoría, en particular los relacionados con el factor de caracterización AMD (UNEP-SETAC Life Cycle Initiative, 2016).

La huella hídrica, definida por Hoekstra a través de la Water Footprint Network, es un indicador que expresa el uso de agua dulce no solo enfocado en el uso directo, sino también en el uso indirecto (Hoekstra, 2017). Se usa para la evaluación de la apropiación de los recursos de agua dulce y se aplica para estimar volúmenes consuntivos en diferentes niveles (Aldaya, 2015). En ese contexto, la huella considera tres componentes la huella azul, la huella verde y la huella gris; la primera de ellas se refiere al consumo de agua dulce, tanto superficial como subterránea, a lo largo de la cadena de suministro, donde el "consumo" se expresa como la pérdida de agua disponible en una zona de estudio, por lo general suele emplear el criterio de cuencas. El segundo componente, la huella verde se refiere al consumo de agua proveniente de fuentes pluviales que no se convierte en escorrentía. Por último, la huella gris está definida como el volumen de agua dulce que se requiere para asimilar y diluir la carga de contaminantes hasta obtener una concentración aceptable compatible con los estándares de calidad establecidos (SABMiller and WWF, 2009).

Cabe señalar que la huella hídrica, empleada como indicador del uso de agua, difiere de las medidas clásicas de "retiros de agua" por tres consideraciones. Primero, al no considerar los volúmenes de agua azul que se devuelven al área delimitada de estudio; al no restringirse, en segundo lugar, únicamente al uso de agua azul, sino tomar en cuenta también al agua verde y gris; y tercero, al no limitarse a los usos directos, sino también a los usos indirectos. Desde esa perspectiva, la huella hídrica ofrece una perspectiva dual, tanto para el consumo como para la producción (CADIS, 2016). Si bien es cierto que, el volumen de consumo de agua puede considerarse como un impacto ambiental, la huella hídrica no es suficiente para determinar la severidad de éste, dado que depende de la vulnerabilidad del sistema o de la cantidad de usuarios o contaminadores vertido (Water Footprint Network, 2011).

La huella hídrica y la huella de agua son herramientas que comparten un objetivo similar, que permite conocer el consumo de agua y sus consecuencias ambientales. Para determinar el consumo de agua de un proceso de producción determinado y tomar decisiones respecto a la asignación de los recursos hídricos para satisfacer la demanda directa e indirecta, resulta más adecuada la metodología de la 
Water Footprint Network (Hoekstra, 2003). Pero, si el objetivo del estudio es determinar el impacto que se produce sobre la disponibilidad de agua o el daño potencial sobre la salud humano y los ecosistemas, el enfoque más apropiado sería la huella de agua (ISO 14046, 2014). Además, es importante considerar el entorno (factores naturales, sociales, culturales, económicos y estéticos) que interactúan entre sí con el individuo y con la comunidad, determinando su forma, carácter, relación y supervivencia (Conesa et al. 2010). En general, se puede decir que el ambiente comprende dos medios: el medio natural y el medio humano (Ferrandis et al. 2006).

En esa línea argumentativa, se debe tomar en cuenta además al sector en el que se aplica la huella hídrica o la huella de agua. Por ejemplo, en la agricultura se puedeclasificar en función de la magnitud de producción, relación con el mercado y dependencia del agua (Larrazábal, 2019). Al describir la agricultura bajo riego, se identifican los sistemas de riego de flujo no controlado y los de flujo controlado (Arteaga et al. 2015), siendo los más utilizados el riego por inundación y por surcos (Cadena, 2012), métodos que poseen baja eficiencia (DGIAR, 2015). De otro lado, se tienen a los sistemas tecnificados, aspersión y goteo (Franco, 2018), ambos se caracterizan por una alta eficiencia (DGIAR, 2015). Sin embargo, el concepto de eficiencia de riego no está formalmente definido, pero es importante para considerar la huella hídrica y la huella de agua, la cual se entiende como la cantidad de agua bien aprovechada por los cultivos durante la irrigación de las tierras, del total de agua captada de cierta fuente (DGIAR, 2015).
Establecer los niveles de eficiencia es importante porque permite conocer qué cantidad de recurso hídrico que se pierde durante el riego y así plantear soluciones que la controlen dentro de los límites aceptables. Conceptualmente, se distinguen varios tipos de eficiencia, por ejemplo, la eficiencia de conducción $\left(\mathrm{Ef}_{\mathrm{C}}\right)$, eficiencia de distribución $\left(\mathrm{Ef}_{\mathrm{D}}\right)$ y eficiencia de aplicación $\left(\mathrm{Ef}_{\mathrm{A}}\right)$. En término ambientales se emplea el concepto de ecoeficiencia, que es una herramienta de gestión para el desarrollo sostenible. Según el CEMDS (1992), el concepto de ecoeficiencia se basa en el ideal de "crear más bienes y servicios haciendo uso de la menor cantidad de recursos y generando la menor cantidad de residuos y contaminantes", tal como lo refiere la norma ISO 14045 de Gestión Ambiental de la Eco-Eficiencia (ISO, 2012). De ese modo, la ecoeficiencia se apoya principalmente en reducir la sobreexplotación de los recursos naturales y disminuir la contaminación asociada a los procesos productivos, es decir, la ecoeficiencia busca un incremento de la productividad de los recursos naturales, así como la reducción de los impactos ambientales a lo largo de todo el ciclo de vida de los productos (Leal, 2005).

Operacionalmente, la ecoeficiencia se expresa como el valor del producto o servicio, representado por la cantidad de bienes producidos o entregados, así como por las ventas netas obtenidas por la comercialización de estos. Por otro lado, la influencia ambiental puede corresponder al consumo de energía, de materiales, de agua, o a las emisiones de gases de efecto invernadero, substancias tóxicas, entre otros. Los indicadores obtenidos se expresan en las unidades relevantes para cada caso, y solo podrán 
ser comparados entre sí o con otros indicadores que provengan de industrias similares y sectores específicos (Zhang y Zhifeng, 2007). Cabe señalar que, al estimar indicadores basados en la huella hídrica extendida es posible determinar la rentabilidad de producción según cultivos (Salmoral et al. 2012). De ese modo, la ecoeficiencia puede ser útil para explicar el uso y consumo del agua, y garantizar su disponibilidad para futuras generaciones (Brundtland, 1987; Viñas, 2012).

\section{Metidología}

\section{Descripción del caso estudio}

El departamento de Lambayeque es un emporio de producción de bienes agrícolas, tanto para consumo nacional como para exportación. Esto ha sido posible gracias a la influencia que ejerce la infraestructura hidráulica. Según el

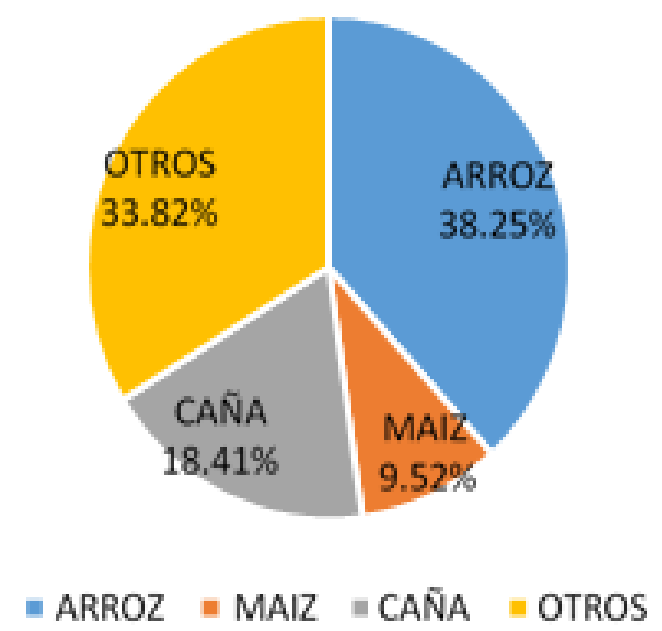

Figura 1-A. Cédula de cultivo de Lambayeque
MINAGRI (2017), el portafolio de cultivos considera en un $55 \%$ de arroz, $15 \%$ de maíz amarillo duro y un 30\% de otros cultivos; en tanto que, en las parcelas de las empresas agroindustriales se siembra en su totalidad caña de azúcar (Figura 1-A). De otro lado, se tiene que el agua utilizada para el riego, en la zona en estudio, proviene de la cuenca del río Chancay-Lambayeque, ubicada en la costa norte del Perú y es una de las más importantes de la vertiente del Pacífico, al abastecer con agua el riego del $87 \%$ de la superficie cultivada de la región Lambayeque. Los principales beneficiarios del recurso hídrico son los productores que conforman la Junta de Usuarios del Valle ChancayLambayeque, compuesta por quince comisiones naturales y tres empresas agroindustriales, abarcando más de cien mil hectáreas bajo riego (JUCHL, 2018), tal como se observa en la Figura 1-B.

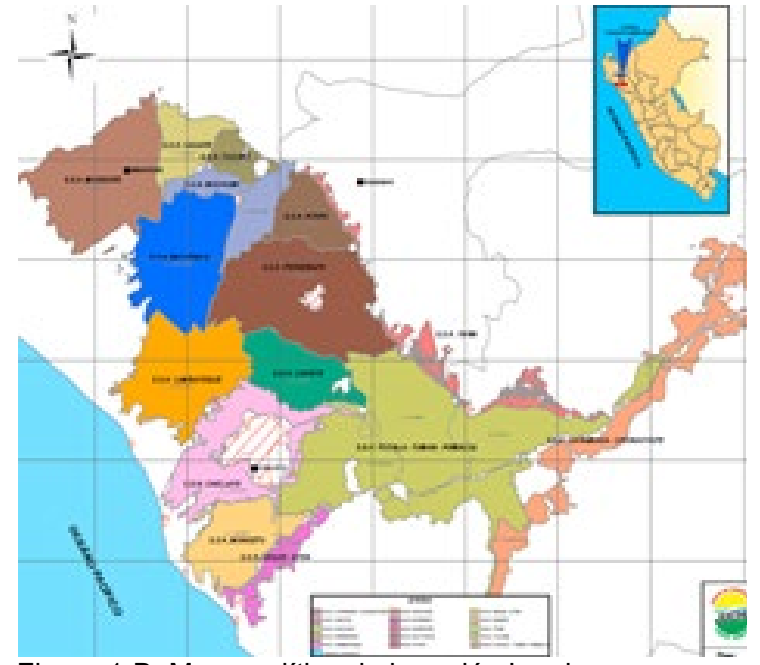

Figura 1-B. Mapa político de la región Lambayeque Figura 1. Cédula de cultivos predominante en el departamento de Lambayeque y mapa político de la región Lambayeque. Fuente: Minagri (2017)

La asignación del agua de la cuenca, administrada por el ANA, varía para cada una de las comisiones, porque se consideran diferentes criterios, tales como: la superficie total a regar y el uso del recurso hídrico. Por la información expuesta en la Tabla 1, se puede suponer que la distribución del agua asignada es proporcional al área sembrada de cada cultivo, aunque deba aceptarse algunas variaciones dependiendo de cada comisión. 
ECoeficiencia de la infraestruCtura hidráUlica del Sistema ChanCay-Lambayeque y su impaCto en la HUELLA HÍDRICA DE LA PRODUCCIÓN AGRÍCOLA

Tabla 1

Caracteristicas centrales de las comisiones de regantes del sistema menor Chancay-Lambayeque

\begin{tabular}{|c|c|c|c|c|c|}
\hline $\begin{array}{l}\text { Comisión de } \\
\text { regantes }\end{array}$ & $\begin{array}{c}\text { Número } \\
\text { de usuarios }\end{array}$ & $\begin{array}{l}\text { Número de } \\
\text { predios }\end{array}$ & $\begin{array}{l}\text { Superficie bajo } \\
\text { riego (ha.) }\end{array}$ & $\begin{array}{l}\text { Volumen asignado } \\
\quad\left(\text { miles de } \mathrm{m}^{3}\right)\end{array}$ & $\begin{array}{c}\text { Dotación }\left(\mathrm{m}^{3} /\right. \\
\text { ha.) }\end{array}$ \\
\hline Sasape & 2405 & 3088 & 5042 & 20280 & 4023 \\
\hline Mochumi & 1831 & 2292 & 4108 & 34465 & 8391 \\
\hline Muy finca & 3339 & 4098 & 10785 & 50263 & 4661 \\
\hline Tucume & 912 & 1114 & 1678 & 11426 & 6812 \\
\hline Morrope & 5244 & 8330 & 12346 & 31527 & 2554 \\
\hline La ramada & 96 & 109 & 314 & 5727 & 18226 \\
\hline Chongoyape & 1865 & 2276 & 10064 & 64743 & 6433 \\
\hline Ferreñafe & 4267 & 5082 & 18136 & 109825 & 6056 \\
\hline Capote & 577 & 903 & 3878 & 29833 & 7693 \\
\hline Lambayeque & 1864 & 2550 & 7448 & 52564 & 7058 \\
\hline Chiclayo & 1733 & 2374 & 7425 & 43515 & 5861 \\
\hline Reque & 726 & 923 & 2028 & 6936 & 3420 \\
\hline Monsefú & 3742 & 4277 & 6446 & 25252 & 3917 \\
\hline Etén & 493 & 622 & 618 & 2112 & 3,420 \\
\hline Pitipo & 723 & 951 & 3707 & 23518 & 6,345 \\
\hline E.A. Pucalá & 1 & 4 & 5997 & 22233 & 3,707 \\
\hline E.A. Pomalca & 1 & 6 & 11153 & 77203 & 6,922 \\
\hline E.A. Tumán & 1 & 4 & 7900 & 36350 & 4,601 \\
\hline Total & 29820 & 39003 & 119069 & 647771 & \\
\hline
\end{tabular}

Nota. Junta de Usuario del sistema menor. Elaboración propia

La asignación de agua y la superficie total bajo riego de cada comisión presentan una distribución similar. Al analizar el indicador de dotación por unidad de superficie, se aprecia que los valores se mantienen en los límites de un promedio de $5500 \mathrm{~m}^{3} / \mathrm{ha}$, a excepción de la comisión de La Ramada, que posee una dotación mayor (18 $000 \mathrm{~m}^{3} / \mathrm{ha}$.); la sobreasignación del recurso se debe a que esta comisión no se encuentra regulada por la junta y posee una cantidad de usuarios y predios que no figuran en los datos públicos; además que en su ámbito existen varias tomas de agua no reguladas que afectan a los volúmenes asignados. Sin embargo, esta condición afecta ligeramente la precisión de los resultados obtenidos, por ello se decidió no considerar un factor de corrección, porque el volumen asignado es inferior al $1 \%$ del agua disponible total. Del lado del consumo, era necesario contar con información sobre los rendimientos promedio de los principales cultivos de la región Lambayeque; la fuente de esa información consultada proviene de la Encuesta Nacional Agropecuaria 2017 (INEI, 2017) y se usó para obtener las toneladas producidas unidad de superficie según comisiones (Tabla 2). 
Tabla 2

Volúmenes de producción y asignación de agua según comisiones del sistema menor ChancayLambayeque

\begin{tabular}{|c|c|c|c|c|c|c|}
\hline $\begin{array}{l}\text { Comisión de } \\
\text { regantes }\end{array}$ & $\begin{array}{l}\text { Producción } \\
\text { Caña (ton) }\end{array}$ & $\begin{array}{l}\text { Producción } \\
\text { Arroz (ton) }\end{array}$ & $\begin{array}{l}\text { Producción } \\
\text { Maíz (ton) }\end{array}$ & $\begin{array}{l}\text { Volumen } \\
\text { asignado } \\
\text { Caña } \\
\left(\text { miles } \mathrm{m}^{3}\right)\end{array}$ & $\begin{array}{l}\text { Volumen } \\
\text { asignado } \\
\text { Arroz } \\
\left(\text { miles m }{ }^{3}\right)\end{array}$ & $\begin{array}{l}\text { Volumen } \\
\text { asignado } \\
\text { Maíz } \\
(\text { miles m³) }\end{array}$ \\
\hline Sasape & & 23826 & 6418 & & 11965 & 3853 \\
\hline Mochumi & & 18096 & 3302 & & 18956 & 4136 \\
\hline Muy finca & & 48377 & 11561 & & 28147 & 8042 \\
\hline Tucume & & 6719 & 1236 & & 5713 & 1257 \\
\hline Morrope & & 51422 & 16543 & & 16394 & 6305 \\
\hline La ramada & & 1258 & 379 & & 2864 & 1031 \\
\hline Chongoyape & & 45143 & 10789 & & 36256 & 10359 \\
\hline Ferreñafe & & 81349 & 20656 & & 61502 & 18670 \\
\hline Capote & & 16773 & 2598 & & 16110 & 2983 \\
\hline Lambayeque & & 34002 & 7484 & & 29961 & 7885 \\
\hline Chiclayo & & 32114 & 5969 & & 23497 & 5222 \\
\hline Reque & & 8122 & 1495 & & 3468 & 762 \\
\hline Monsefu & & 28915 & 8638 & & 14141 & 5050 \\
\hline Eten & & 2820 & 579 & & 1204 & 296 \\
\hline Pitipo & & 16627 & 3974 & & 13170 & 3763 \\
\hline E.a. pucala & 442424 & & & 22233 & & \\
\hline E.a. pomalca & 663513 & & & 77202 & & \\
\hline E.a. tumán & 565338 & & & 36350 & & \\
\hline Total & 1671275 & 415561 & 101622 & 135785 & 283349 & 79615 \\
\hline
\end{tabular}

Nota. Junta de usuario del sistema menor Chancay-Lambayeque e INEI (2017).

\section{Alcance y objetivos}

El objetivo principal consistió en determinar la huella hídrica y estimar la ecoeficiencia de los cultivos más importantes de la región Lambayeque, con base en la eficiencia de la infraestructura hidráulica y de ese modo diseñar políticas de mejora de la productividad en el uso del agua y el suelo para la producción agrícola. En congruencia con este objetivo central, se consideró como objetivos específicos los siguientes: a) Estimar y comparar huellas hídricas reales y teóricas de los cultivos más relevantes de la zona de estudio, con uso del software CROPWAT $8.0 \mathrm{y}$ b) estimar la ecoeficiencia, a través del cálculo de indicadores, que relacionen el valor económico generado por el producto respecto a la cantidad de recursos consumidos para su producción y determinar las comisiones que tengan una mejor relación respecto al promedio.

\section{Cálculo de la huella hídrica teórica}

Para el cálculo de las huellas hídricas se empleó el software CROPWAT 8.0 (FAO, 2019). El modelado se complementó con la base de datos CLIMWAT 2.0 (FAO, 2019), que reúne información meteorológica de diversas estaciones climáticas alrededor del 
mundo; proporcionando datos promedio mensuales de precipitación, velocidad del viento, temperaturas mínimas y máximas, horas de insolación y humedad. El modelado consideró estudios previos, donde se realizó el cálculo de la huella hídrica de cultivos (Renderos, 2014, Bolaños, 2011), siguiendo los lineamientos metodológicos de la Water Footprint Network, es decir, se empleó la función RAC (Requerimiento de Agua del Cultivo) del CROPWAT 8.0, que utiliza un modelo que calcula la evapotranspiración del cultivo en condiciones de crecimiento ideal desde la siembra hasta la cosecha, asumiendo el requerimiento de agua del cultivo bajo circunstancias particulares de su entorno, la información sobre la precipitación efectiva que recibe el cultivo y los requerimientos de irrigación para evitar cualquier tipo de limitación durante el periodo vegetativo del cultivo.

\section{Descripción de la herramienta}

Como se dijo anteriormente, se empleó el software CROPWAT 8.0 en sus cinco módulos, de los cuales cuatro definen los datos de entrada, en tanto que el quinto permite la obtención de datos de salida, conforme al detalle que se explica en la Tabla 3.

Tabla 3

Detalle de los módulos del software CROPWAT 8.0 empleado en el modelado de la Huella Hidrica de los cultivos del ámbito del sistema menor Chancay-Lambayeque

\begin{tabular}{|c|c|c|c|}
\hline Módulo & Descripción & Comentario & Fuente de datos \\
\hline $\mathrm{Clima}_{\mathrm{ET}}$ & $\begin{array}{l}\text { Se considera los parámetros medios mensuales de } \\
\text { temperatura mínima }\left({ }^{\circ} \mathrm{C}\right) \text {, temperatura máxima } \\
\left({ }^{\circ} \mathrm{C}\right) \text {, humedad }(\%) \text {, velocidad del viento }(\mathrm{km} / \\
\text { día) e insolación (horas). Utiliza los datos propor- } \\
\text { cionados para el cálculo de la radiación }\left(\mathrm{MJ} / \mathrm{m}^{2} /\right. \\
\text { día) y la evapotranspiración de referencia }(\mathrm{mm} / \\
\text { día), mediante la aplicación del método de Pen- } \\
\text { man-Monteith. }\end{array}$ & $\begin{array}{l}\text { Los datos a introducir en este módulo } \\
\text { son extraídos del CLIMWAT 2.0, espe- } \\
\text { cíficamente de las estaciones selecciona- } \\
\text { das para la zona de estudio. }\end{array}$ & $\begin{array}{l}\text { Datos extraídos de la es- } \\
\text { tación } \# 45 \text { del Perú, ubi- } \\
\text { cada en el departamento } \\
\text { de Lambayeque a una } \\
\text { altura de } 27 \text { msnm, la- } \\
\text { titud } 6.68^{\circ} \text { y longitud } \\
79.88^{\circ} \text {. Corresponde a la } \\
\text { base de datos del software } \\
\text { CLIMWAT } 2.0 \text {. }\end{array}$ \\
\hline Precipitación & $\begin{array}{l}\text { Se requiere valores de precipitación }(\mathrm{mm}) \text { que pue- } \\
\text { den ser diarios, decadiarios o mensuales, los cuales } \\
\text { también son extraídos de las estaciones específicas } \\
\text { seleccionadas haciendo uso del CLIMWAT 2.0. }\end{array}$ & $\begin{array}{l}\text { Se calcula la precipitación efectiva con } \\
\text { el método de la USCS (United State } \\
\text { Soil Conservation Service). }\end{array}$ & \\
\hline Cultivo & $\begin{array}{l}\text { Se requiere valores específicos para cada cultivo, } \\
\text { como el } \mathrm{K}_{\mathrm{c}} \text { (adimensional), duración de las etapas } \\
\text { de desarrollo (días), profundidad de las raíces }(\mathrm{m}) \text {, } \\
\text { agotamiento crítico (fracción), respuesta del ren- } \\
\text { dimiento (fracción) y opcionalmente, la altura del } \\
\text { cultivo (m). También se debe ingresar la fecha de } \\
\text { siembra para que el software identifique los datos } \\
\text { climáticos utilizar. }\end{array}$ & $\begin{array}{l}\text { El software posee archivos predetermi- } \\
\text { nados para varios cultivos (cańa de azú- } \\
\text { car, arroz y maíz; cambiando las fechas } \\
\text { de siembra para la zona de estudio. }\end{array}$ & $\begin{array}{l}\text { Base de datos en CRO- } \\
\text { PWAT } 8.0 \text {, para los culti- } \\
\text { vos de la cańa de azúcar, el } \\
\text { arroz y el maíz. }\end{array}$ \\
\hline Suelo & $\begin{array}{l}\text { Considera parámetros de humedad del suelo dis- } \\
\text { ponible total }(\mathrm{mm} / \mathrm{m}) \text {, tasa máxima de infiltra- } \\
\text { ción de la precipitación }(\mathrm{mm} / \text { día }) \text {, profundidad } \\
\text { radicular máxima }(\mathrm{cm}) \text {, agotamiento inicial de la } \\
\text { humedad de suelo }(\%) \text { y la humedad de suelo ini- } \\
\text { cialmente disponible }(\mathrm{mm} / \mathrm{m}) \text {. }\end{array}$ & $\begin{array}{l}\text { Para el caso del arroz, debido al riego } \\
\text { por inundación, se requiere sobre la po- } \\
\text { rosidad drenable (\%), el agotamiento } \\
\text { crítico para grietas del fangueo (frac- } \\
\text { ción), la tasa máxima de percolación } \\
\text { después del fangueo ( } \mathrm{mm} / \text { día), la dis- } \\
\text { ponibilidad de agua a la siembra (mm) } \\
\text { y la altura máxima de la lámina de agua } \\
(\mathrm{mm}) \text {. }\end{array}$ & $\begin{array}{l}\text { Base de datos en el softwa- } \\
\text { re CROPWAT 8.0, para } \\
\text { el tipo de suelo rojo fran- } \\
\text { co-arenoso, que es el que } \\
\text { más se asemeja al de la zona } \\
\text { de estudio. }\end{array}$ \\
\hline $\begin{array}{l}\text { Requerimien- } \\
\text { to de Agua del } \\
\text { Cultivo (RAC) }\end{array}$ & $\begin{array}{l}\text { Proporciona los datos de salida, como la evapo- } \\
\text { transpiración del cultivo ( } \mathrm{mm}) \text {, precipitación } \\
\text { efectiva }(\mathrm{mm}) \text { y requerimientos de riego }(\mathrm{mm}) \text {; } \\
\text { que posteriormente se emplea para el cálculo de } \\
\text { huellas hídricas. }\end{array}$ & $\begin{array}{l}\text { Los valores están calculados para perio- } \\
\text { dos de diez días, es decir, en un régimen } \\
\text { decadiario; siendo la unidad de referen- } \\
\text { cia final la de } \mathrm{mm} / \mathrm{dec} \text {. }\end{array}$ & \\
\hline
\end{tabular}




\section{Modelo matemático}

El cálculo de huella hídrica consideró la siguiente ecuación:

$$
H H_{i}=\frac{R A C_{i}}{Y}
$$

Donde, $\mathrm{HH}$ representa la magnitud de la huella hídrica para un cultivo específico expresada en unidades de volumen sobre masa, "i" puede tomar el valor del componente "verde" o "azul" de la huella. RAC, es el requerimiento de agua del cultivo, bien sea verde o azul, y su unidad está expresada en volumen por área, Y representa el rendimiento del cultivo en unidades de masa por área.

Para calcular el RAC, se deben sumar los valores de la evapotranspiración real del cultivo $\mathrm{ET}_{\mathrm{c}}$, a lo largo del periodo vegetativo $(\mathrm{d}=1 \rightarrow \mathrm{d}=\mathrm{n})$, tal y como se muestra en la siguiente ecuación:

$$
R A C_{i}=10 * \sum_{d=1}^{d=n} E T_{c, i}
$$

Donde, ET ${ }_{c}$ está expresada en milímetros día ${ }^{-1}$ y el factor 10 se emplea para la conversión a metros cúbicos sobre hectáreas. Los valores de $\mathrm{ET}_{\mathrm{c}}$ son obtenidos directamente del software, a través del método Penman-Monteith.
Por último, se debe establecer la diferencia entre la evapotranspiración verde del cultivo ET $_{\text {verde }}$ y la evapotranspiración azul del cultivo $\mathrm{ET}_{\text {azul }}$. El componente verde corresponde al valor mínimo de la evapotranspiración del cultivo y la precipitación efectiva y el componente azul corresponde al valor máximo entre cero y la diferencia entre la evapotranspiración del cultivo y la precipitación efectiva, conforme a las ecuaciones siguientes:

$$
\begin{aligned}
& E T_{\text {verde }}=\min \left(E T_{c}, P_{\text {ef }}\right) \\
& E T_{a z u l}=\max \left(E T_{c}-P_{e f}, 0\right)
\end{aligned}
$$

\section{Indicadores y estimación gráfica de ecoeficiencia}

Zhang y Zhifeng (2007) consideran que al evaluar cualquier sistema productivo o de servicios se presenta dos tipos de eficiencia: una técnica y otra ambiental. En agricultura se cuenta con el indicador de la huella hídrica extendida, que pretende realizar una valoración económica del agua para conocer si el consumo resulta ser una inversión adecuada. Un estudio realizado por Salmoral et al. (2012), presenta el diseño conceptual del indicador de $\mathrm{HH}$ extendida (Figura 2).

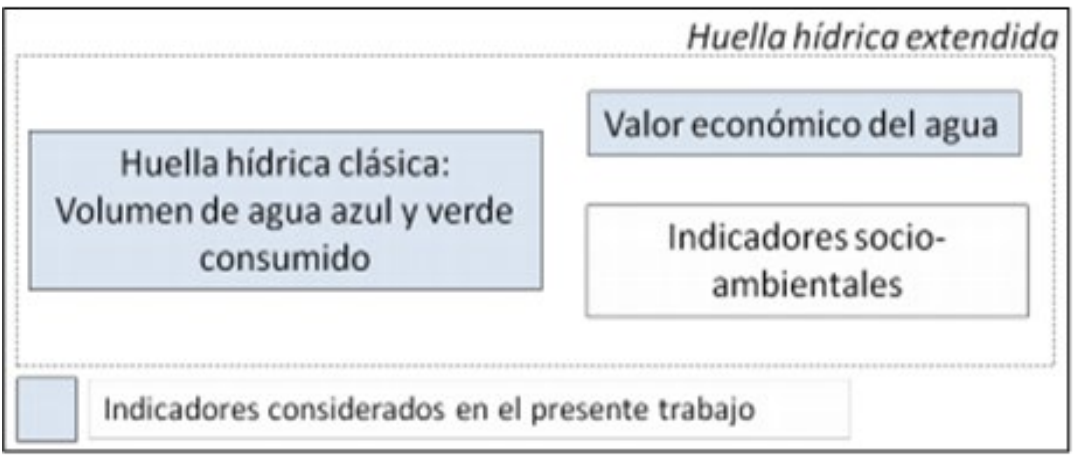

Figura 2. Bases conceptuales del indicador huella hídrica extendida.

Fuente: Salmoral el al (2012) 
Los indicadores elegidos fueron la Productividad Aparente del Agua y la Productividad Aparente del Suelo (AWP y ALP) respectivamente, por sus siglas en inglés. La Productividad Aparente del Agua (AWP) se utilizó para estimar el valor de la producción agrícola en nuevos soles por unidad de agua consumida y en su cálculo se usó la siguiente expresión:

$$
A W P_{i}=\frac{P_{m, i}}{H H_{A, i}}
$$

Donde, $\mathrm{P}_{\mathrm{m}}$ es el precio de mercado del producto cultivado en nuevos soles por tonelada, y $\mathrm{HH}_{\mathrm{A}}$ representa la huella hídrica azul en metros cúbicos por tonelada; siendo "i" una variable que puede tomar el valor de cualquier cultivo analizado (cańa de azúcar, el arroz o el maíz). Por otro lado, la Productividad Aparente del Suelo (ALP) se empleó para estimar el valor económico generado por hectárea de tierra cultivada, con la siguiente ecuación:

$$
A L P_{i}=P_{m, i} \times Y_{i}
$$

Donde, $\mathrm{P}_{\mathrm{m}}$ es el precio de mercado del producto cultivado en nuevos soles por tonelada, y Y es el rendimiento del cultivo en toneladas por hectárea cultivada; siendo "i" una variable que representa el cultivo sobre el cuál se calcula los indicadores.

El indicador de ecoeficiencia y su ecuación matemática, relaciona el valor de la producción con la influencia ambiental ocasionada por esta. Para ello, se empleó la producción neta de un cultivo específico para representar el valor y el volumen neto de agua consumida para su producción, que expresa el impacto sobre el ambiente. Primero, se estandarizó los valores calculadosde producción yagua consumida para cada comisión, con recorrido de 0 a 1 , a fin de lograr comparaciones múltiples. Luego, se estableció pares ordenados que representan la relación de eficiencia y se graficó, de manera que se pueda determinar qué comisiones poseen una mejor relación entre insumo y recurso (Figura 3); donde los pares ordenados ubicados por encima de la diagonal son más ecoeficientes debido a altas tasas de producción con bajo consumo de agua, mientras que para los que están debajo resultan ser ecoineficientes.

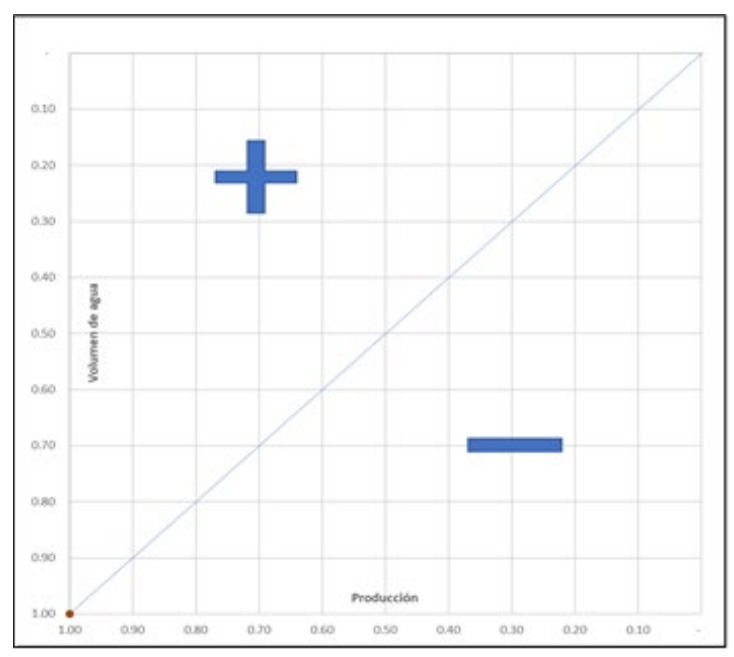

Figura 3. Diagrama de ecoeficiencia con indicadores adimensionales (ambiental y económico)

\section{Resultados}

\section{Huellas hídricas en el contexto del sistema menor Chancay-Lambayeque}

Como se puede apreciar en las Figuras 4-A y 4-B, que la relación insumo/producto entre cultivos varía dependiendo de las unidades en los que se representen, debido al efecto que ejerce el concepto de rendimiento de producción. También se aprecia que la huella hídrica verde no es significativa, porque como verá más adelante, representa una fracción marginal de la huella hídrica total según cultivos. 


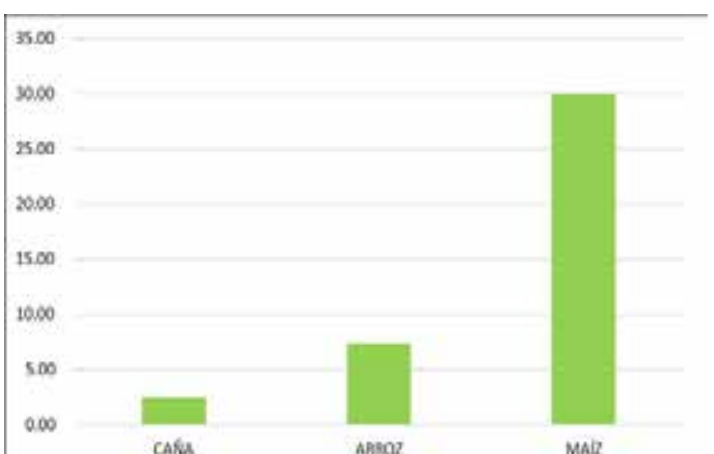

Figura 4-A. Huella hídrica verde según volumen de producción

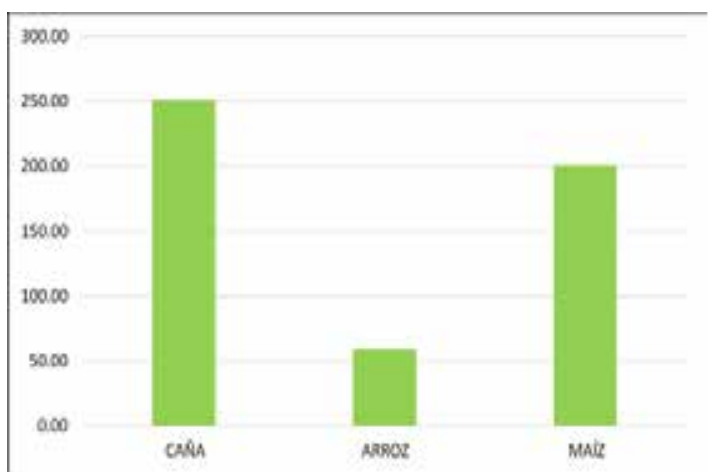

Figura 4-B. Huella hídrica verde según superficie

Figura 4. Huella hídrica verde por volumen de producción $\left(\mathrm{m} 3 \cdot \mathrm{ton}^{-1}\right)$ y superficie $\left(\mathrm{m} 3 \cdot \mathrm{ha}^{-1}\right)$ según principales cultivos del sistema menor Chancay-Lambayeque

En el caso de la huella hídrica azul expresada por el volumen total de agua de riego que se incorporó al producto, se tiene que el arroz presenta una mayor huella hídrica al requerirse agua para la fase de preparación del suelo con riego posterior por inundación, en esta operación se utilizan alrededor de $370 \mathrm{~m}^{3} \cdot$ ton $^{-1}$; práctica agronómica que explica el incremento sustancial de la huella hídrica del arroz (Figura 5-A). De otro lado, en cuanto a los volúmenes

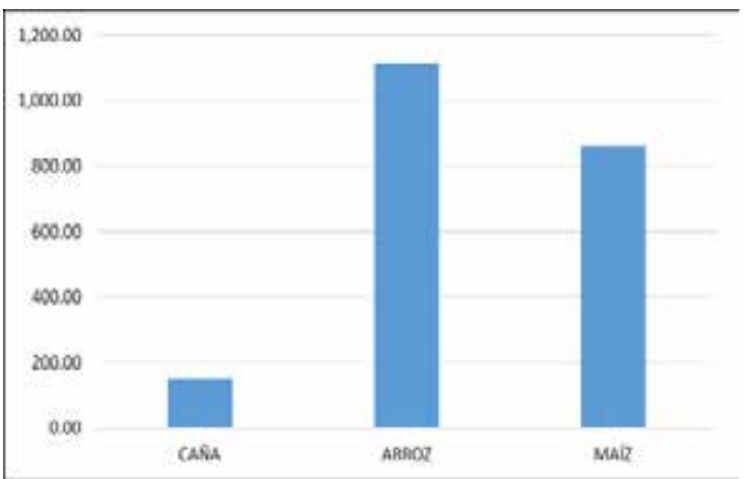

Figura 5-A. Huella hídrica azul según volumen de producción de agua por unidad de superficie, se aprecia que la cańa de azúcar es el cultivo con mayor huella (Figura 5-B). Sin embargo, se debe considerar que CROPWAT 8.0 solo calcula la cantidad de agua requerida por el cultivo, más no el total de agua utilizada, que depende de la eficiencia de riego y de las pérdidas por distribución, esto se debe tomar en cuenta para corregir el valor de la HHA al realizar las estimaciones de indicadores económicos.

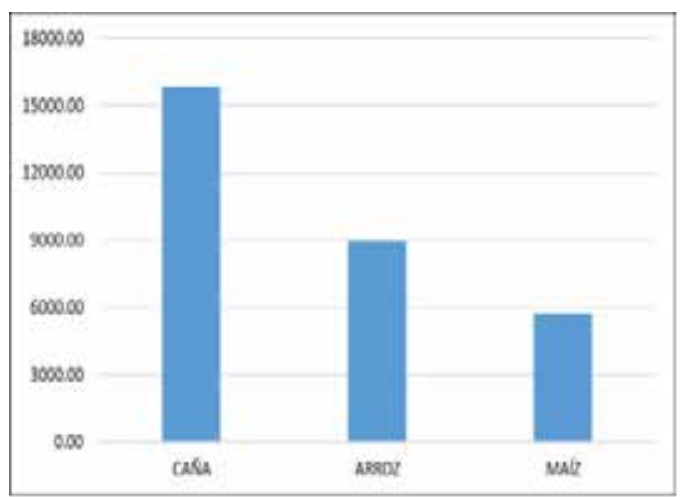

Figura 5-B. Huella hídrica azul según superficie

Figura 5. Huella hídrica azul por volumen de producción $\left(\mathrm{m}^{3} \cdot \operatorname{ton}^{-1}\right)$ y superficie $\left(\mathrm{m}^{3} \cdot \mathrm{ha}^{-1}\right)$ según principales cultivos del sistema menor Chancay-Lambayeque

Al integrar la huella verde y azul se obtiene la huella hídrica total, la cual considera un componente adicional: la huella hídrica gris; la cual no se calculó para los fines prácticos del estudio. La huella hídrica total, expresada gráficamente en intensidades de uso para cada cultivo evaluado (Figuras 5-A, 5-B, 5-C, 5-D, 5-E y 5-F) y muestran la naturaleza marginal en la contribución para la formación de biomasa en todos los 
cultivos, pero en caso del arroz, es nula debido a que suele sembrarse en periodos del año con escasa precipitación. De ese modo, se puede ver que la huella hídrica verde (Figura 5-A), sin importar la unidad en la que se exprese, representa en promedio

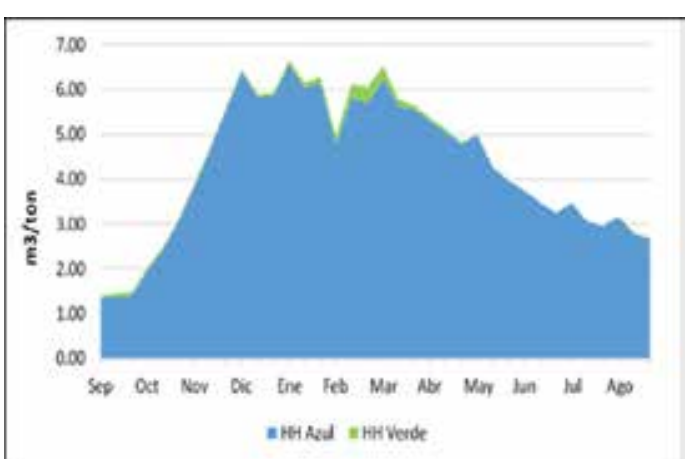

Figura 6-A. Huella hídrica total del cultivo de caña azúcar.

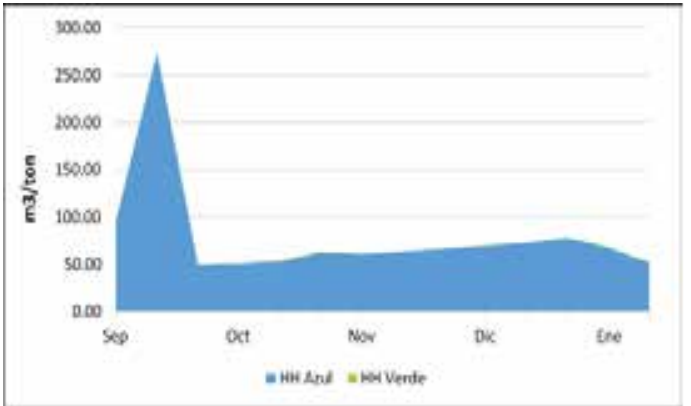

Figura 6-C. Huella hídrica total del cultivo de arroz.

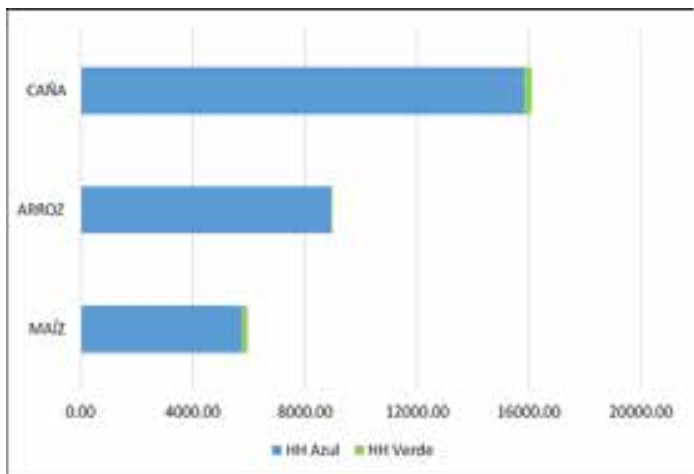

Figura 6-D. Huella hídrica total según cultivos en $\mathrm{m}^{3}$.ha-1 el $1.86 \%$ de la huella hídrica total, debido a los bajos niveles de precipitación en la zona de estudio. Por tanto, este aspecto influye en la disminución de la eficiencia de producción porque obliga a consumir más agua azul a través del riego.

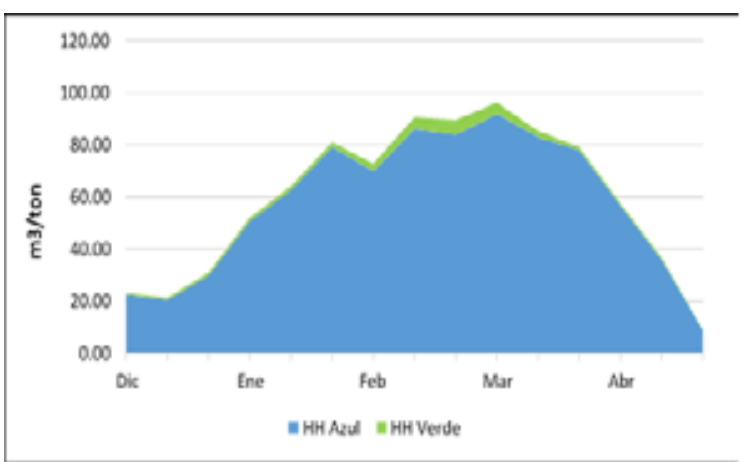

Figura 6-B. Huella hídrica total del cultivo de maíz.

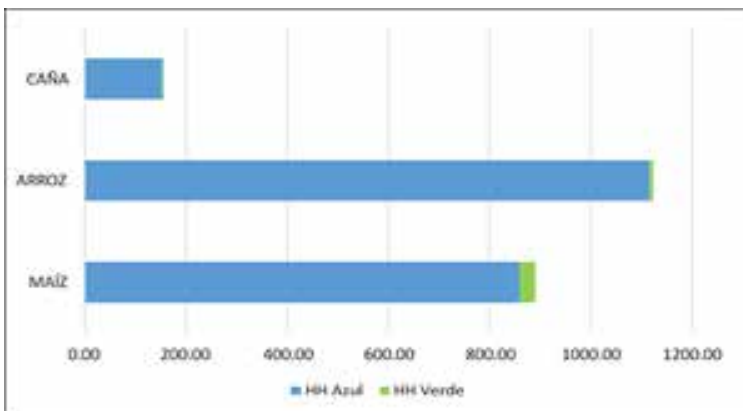

Figura 6-D. Huella hídrica total según cultivos en $\mathrm{m}^{3} \cdot$ ton $^{-1}$

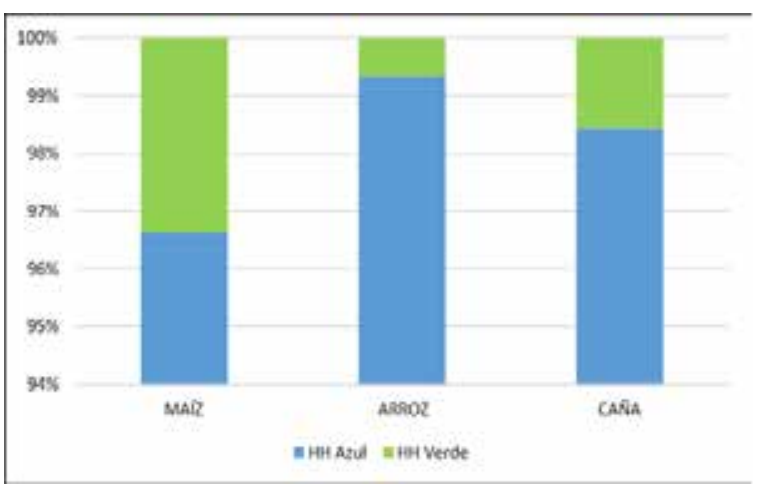

Figura 6-D. Composición de la Huella hídrica total según cultivos en porcentaje

Figura 6. Huella hídrica total por volumen de producción $\left(\mathrm{m}^{3} \cdot \operatorname{ton}^{-1}\right)$ y superficie $\left(\mathrm{m}^{3} \cdot \mathrm{ha}^{-1}\right)$ según principales cultivos del sistema menor Chancay-Lambayeque

Comparación entre la huella hídrica real y la teórica: discutiendo la incertidumbre del modelado

$\mathrm{Al}$ conocerse los volúmenes reales incorporados a los cultivos, se puede convertir la información estimada en una "huella hídrica real", esta huella además debe promediarse a nivel de todas las comisiones y de cada cultivo, comparándose con la huella hídrica teórica. La Figura 7 describe la brecha 
existente entre huellas y se aprecia que los valores reales son menores a los calculados por simulación, lo que sugiere que se utiliza menos agua de la que teóricamente se necesita. En ese sentido, las discrepancias halladas se deben a tres factores. El primero, el conservador factor empleado en el cálculo de pérdidas y por otro lado, la asunción de porcentajes de pérdida altos, que de acuerdo con la literatura y mediciones empíricas, no deberían estar lejos de los valores verdaderos. El segundo factor se debe a los datos empleados, que considera únicamente los volúmenes facturados reconocidos por los usuarios, pero que en realidad solo representan una fracción del agua total consumida, mientras que la diferencia podría ser catalogada como "sustracción". El tercer factor tiene que ver con el concepto de riego deficitario, que pudiera ser beneficioso al priorizar el rendimiento del recurso, en función del rendimiento del cultivo.

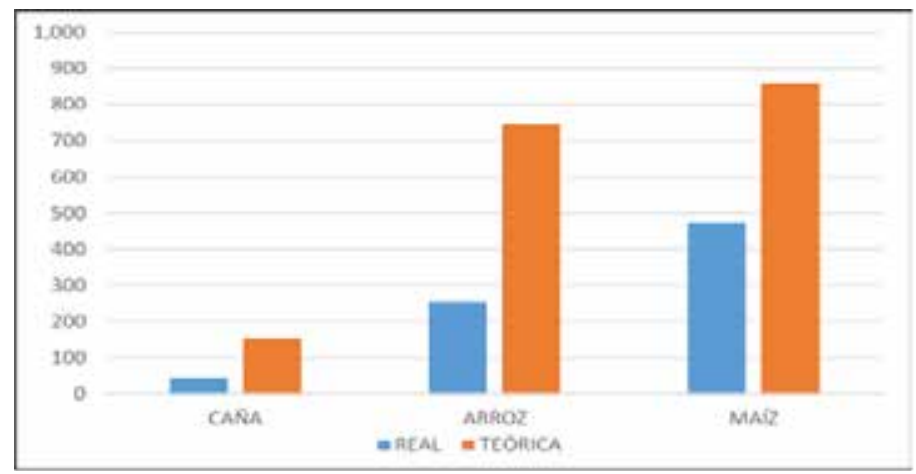

Figura 7. Huellas hídricas totales reales y teóricas o modeladas según cultivos en $\mathrm{m}^{3}$.ton ${ }^{-1}$

\section{Ecoeficiencia de los cultivos}

Una forma de conocer si los recursos naturales como el agua y el suelo, en este caso, son aprovechados de manera óptima para la producción agrícola, es por medio de la estimación de la ecoeficiencia, como se argumentó líneas arriba, con base en los datos de la Encuesta Nacional Agraria, que se detallan en la Tabla 4.

Tabla 4

Valores asumidos de AWP y ALP según cultivos para la estimación de la ecoeficiencia

\begin{tabular}{lrrr}
\hline & Cańa de azúcar & \multicolumn{1}{c}{ Arroz } & Maíz amarillo duro \\
\hline Rendimiento (ton/ha) & 103,44 & 8,01 & 6,70 \\
Valor mercado (S/./ton) & 78,40 & 1295,90 & 919,30 \\
HH azul real (m ${ }^{3} /$ ton$)$ & 45,00 & 254,00 & 473,00 \\
HH azul real $\left(\mathrm{m}^{3} / \mathrm{ha}\right)$ & 465480 & 2034,54 & 3169,10 \\
\hline AWP $\left(\mathrm{S} / \mathrm{m}^{3}\right)$ & 1,74 & 5102 & 1,94 \\
ALP $(\mathrm{S} / \mathrm{ha})$ & 8109,70 & 10380,16 & 6159,31 \\
\hline
\end{tabular}

Nota. Encuesta Nacional Agraria 2017 (INEI, 2017)

Al comparar los valores de AWP, como se muestra en la Figura $8-A$, se aprecia que el arroz posee la mejor relación en cuanto al valor monetario obtenido por su comercialización respecto al agua consumida para su producción. Esto indicaría que es económicamente eficiente en términos de retorno por el recurso 
hídrico y que, a pesar de tener métodos de riego poco eficaces, su valor de mercado retribuye mejor, en términos relativo, el abundante consumo de agua. Sin embargo, esta apreciación solo considera la eficiencia técnica más no la ambiental. Ahora, respecto a los valores ALP, Figura $8-B$, indican que el arroz también es el

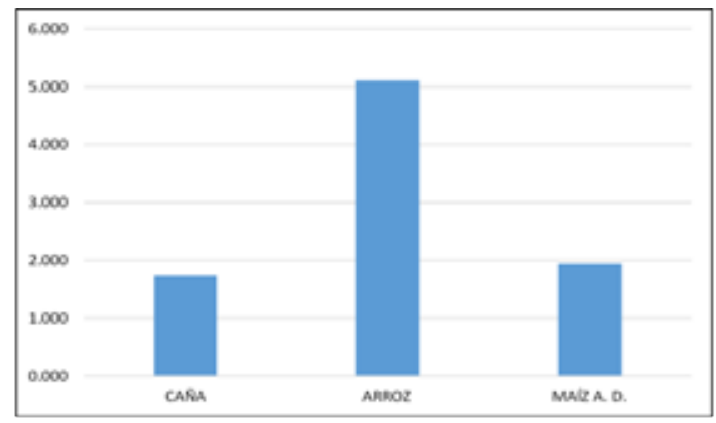

Figura 8-A. Productividad aparente del agua por cultivos en soles por $\mathrm{m}^{3}$ cultivo con mayor eficiencia económica en cuanto a su superficie. Esto significa que genera una mayor contribución en los ingresos monetarios por hectárea en comparación con los demás cultivos y, por lo tanto, una parcela de arroz posee mayor valor comercial que una de cańa de azúcar o maíz amarillo duro.

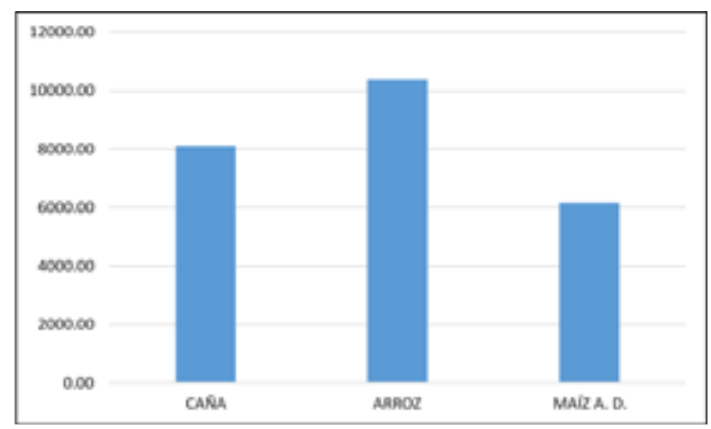

Figura 8-B. Productividad aparente del suelo según cultivos en soles por ha.

Figura 8. Productividad aparente del agua según cultivos expresada en soles por volumen $\left(\mathrm{m}^{3}\right)$ de agua empleada y productividad aparente del suelo según cultivos en soles por unidad de superficie (ha).

Al comparar la ecoeficiencia relativa, entre comisiones, se aprecia en el caso del arroz (Figura 9-A), que la comisión más ecoeficiente es Morrope porque, en comparación con el resto, utiliza menores volúmenes de agua para lograr niveles de producción altos. De manera opuesta, la comisión menos ecoeficiente es Mochumi, que se encuentra más alejada de la línea de

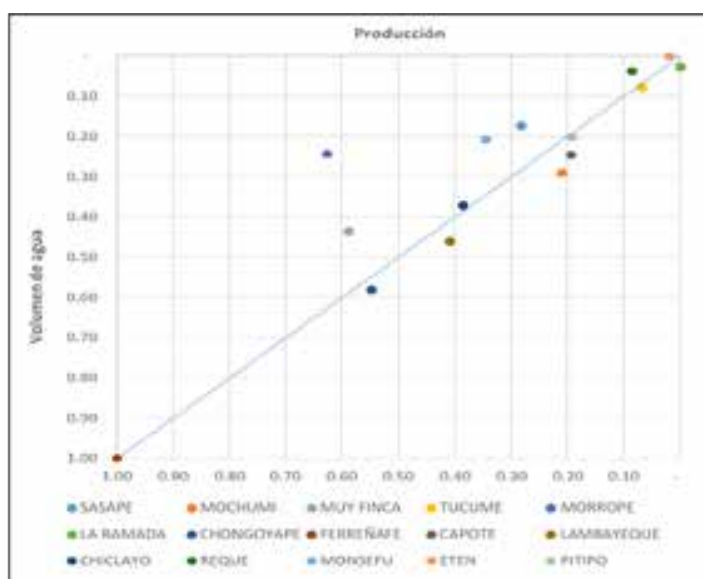

Figura 9-A. Ecoeficiencia comparada para el cultivo de arroz según comisiones del sistema menor ChancayLambayeque proporcionalidad teórica. En el caso del maíz (Figura 9-B), se observa que la comisión más ecoeficiente también es Morrope, mientras que las demás se mantienen cercanas al promedio. Cabe señalar que este análisis no se realizó para el cultivo de la caña, debido a que solo se cultiva, en grandes extensiones, en tres comisiones y las estimaciones podrían ser no representativas.

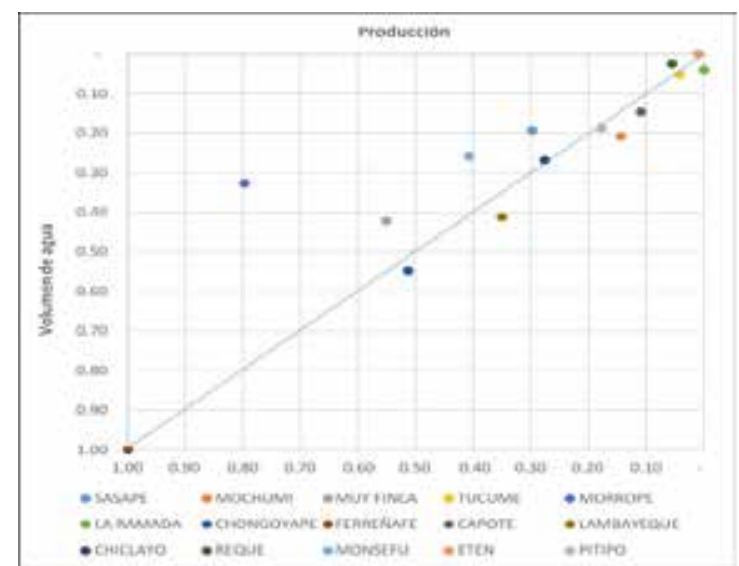

Figura 9-B. Ecoeficiencia comparada para el cultivo de maíz según comisiones del sistema menor ChancayLambayeque

Figura 9. Ecoeficiencias comparadas para el caso de los cultivos de arroz y maíz según comisiones del sistema menor Chancay-Lambayeque 


\section{Discusión}

\section{Huella hídrica teórica y real en cultivos intensivos}

Existen varios estudios sobre la huella hídrica teórica con del uso de software CROPWAT, uno de ellos es el realizado por Mekonnen y Hoesktra (2011), donde se estimó huellas hídricas promedio, a nivel mundial, para varios cultivos, en $\mathrm{m}^{3} \cdot$ ton $^{-1}$ de 196, 1487 y 1028 para caña de azúcar, arroz y el maíz respectivamente, valores cercanos a los calculados en el presente estudio, al ser 156 (caña de azúcar), 1 124 (arroz) y $891 \mathrm{~m}^{3} \cdot$ ton $^{-1}$ (maíz). Las variaciones se deben principalmente a las diferencias en los rendimientos considerados, periodos vegetativos, factores climáticos, entre otros; que generan cambios en la evapotranspiración, principal parámetro para estimar los requerimientos hídricos finales. Sin embargo, la principal diferencia se da en la composición de la huella teórica total, porque, al dividirla en sus componentes, verde y azul, se aprecia que la huella verde contribuye en promedio con el $80 \%$ del total de los tres cultivos analizados, mientras que, en este caso, solo representa el 2\% de la huella total. Esto se explica porque la huella verde depende del volumen de precipitaciones, pero en el departamento de Lambayeque las precipitaciones son prácticamente nulas, necesidad que debe ser suplida mediante riego. Por otro lado, al ser la cańa de azúcar y el maíz los cultivos más frecuentes en las zonas tropicales, es consistente que se encuentran enormes superficies bajo el sistema agricultura en secano, aumentando los promedios mundiales de la huella hídrica verde.

En el estudio de Bolaños (2011) en Honduras, se estimó la huella hídrica de quince cultivos, obteniendo 210, 2587 y $1725 \mathrm{~m}^{3} \cdot$ ton $^{-1}$ para cańa de azúcar, arroz y el maíz, respectivamente. El aporte de la huella verde es en promedio del $65 \%$ y para el caso del arroz y el maíz, la huella hídrica es $70 \%$, porcentaje que excede a los promedios mundiales; debido a que Honduras es un país tropical con altas temperaturas y elevados niveles de evapotranspiración, aumentan la demanda de agua requeridos por los cultivos. Por otro lado, la huella hídrica real que resultó ser bastante menor que la teórica, es importante considerar que el software CROPWAT determina los requerimientos hídricos para un desarrollo óptimo del cultivo, aunque el riego sea realizado con cierto nivel de déficit o riego deficitario y se aplica mayormente en las regiones donde los recursos hídricos son limitados, debido a que puede ser más beneficioso maximizar la productividad del agua en lugar de maximizar la cosecha por unidad de tierra (Fereres et al. 2007). Al considerar una estrategia de optimización que postule irrigar los cultivos solo durante las fases sensibles a la sequía, a pesar del estrés en la planta y, por lo tanto, aceptar la pérdida consecuente de producción (Geerts et al. 2009).

Otro factor que acentúa la diferencia entre huellas es el "robo" o "sustracción", que en la teoría de la economía institucional es conocida como el efecto "polizon" o free rider, a ello se añade las pérdidas físicas $\mathrm{u}$ operacionales (Williamson, 2002). En el Perú un 30\% del agua asignada para irrigación agrícola es realmente utilizada, mientras que lo demás se pierde principalmente debido a infraestructura hidráulica precaria $y$ una cultura social y ambiental que favorece robos o sustracciones (ANA, 
2017). En este estudio se estimó un porcentaje promedio de pérdidas de $57.9 \%$, se puede asumir que las pérdidas generadas por robos estarían en 12.1\%. En ese sentido, Trivelli, Escobal y Revesz señalan que, si bien las juntas de usuarios y comisiones de riego reportan beneficios teóricos, en cuanto a la programación y asignación de agua entre los diferentes sectores y cultivos, en la práctica, la distribución de agua no es equitativa porque existen grupos que se ubican en la cabeza de los canales y consumen una mayor cantidad de agua de la que pagan. Similar comportamiento se observa en otros agricultores como una forma de adelantarse a la escasez; además hay quienes roban agua y no son penalizados, y si lo son, no reciben sanciones que desincentiven este tipo de accionar (Trivelli et al. 2006).

\section{Ecoeficiencia de producción de los cultivos intensivos}

Los indicadores económicos estimados permiten concluir que el cultivo más rentable en cuanto al uso del agua es el maíz, mientras que el más rentable respecto al uso del suelo, es el arroz. Sin embargo, estos resultados no se pueden tomar por separado, porque como sostiene Bossio (2010), la clave para un efectivo uso de los recursos en la agricultura es comprender que en el ciclo del agua y el manejo del suelo se encuentran relacionado $y$, por tanto, cualquier incremento en la productividad agrícola del agua solo pueden ser obtenidos con mejoras en el manejo del uso de los suelos. Desde dicha perspectiva, se puede rescatar que el cultivo mejor balanceado es el arroz, porque además de ser el más ecoeficiente en el uso de los suelos, posee un gran margen de mejora en cuanto al uso de agua, porque su eficiencia de riego promedio es considerablemente más baja que otros cultivos. Si se establecieran sistemas con alta eficiencia para su riego, el arroz se convertiría en el cultivo más rentable de la región, debido a que generaría la mayor cantidad de ingresos económicos con respecto a la cantidad de recursos consumidos.

Un estudio realizado reporta que se pueden obtener eficiencias de hasta 3.11 $\mathrm{m}^{3}$ de agua consumida por kilogramo de arroz producido, asumiendo un rendimiento de 6.49 toneladas por hectárea, y solo considerando el agua consumida para el riego por aspersión, mas no la perdida durante su distribución (Benavides et al. 2014). En el presente caso, la eficiencia del arroz es de 3.24 $\mathrm{m}^{3} \cdot$ ton $^{-1}$ de agua por volumen producido, incluso con un rendimiento bajo de los sistemas de infraestructura de manejo de agua. Si fuera posible mejorar los sistemas de distribución y riego sin afectar la producción, los valores de eficiencia serían aún mejores que los reportados en el estudio citado.

Los indicadores AWP y ALP se circunscriben al valor de mercado de los productos y su relación con el consumo de agua generado, ignorando otros impactos en el ambiente, como la contaminación, afectación en la salud y la degradación de los ecosistemas, entre otros. Aun así, son indicadores que pueden utilizarse como referencia para la estimación de la ecoeficiencia, al relacionar el valor del producto con su influencia ambiental, que en este caso es el consumo de recursos. Además, contar con una perspectiva económica permite corroborar la relación intrínseca entre agricultura, recursos naturales y 
economía; argumentando que el acceso a una fuente de agua confiable y sostenible mejora la producción de los cultivos, intensifica las oportunidades de empleo y estabiliza los ingresos y el consumo (De Fraiture et al. 2010).

Respecto a los resultados estimados de la ecoeficiencia a nivel de comisiones, para los casos del arroz y el maíz, la comisión de Morrope presenta un una relación entre volumen de producción y volumen de agua de 2.57 y 2.52 en comparación con el resto, que tienen en promedio ratios de 1.25 y 1.21 para el arroz y el maíz, respectivamente. Esto quiere decir que produce el doble de las demás comisiones con la misma cantidad de agua, lo cual es coherente al presentar la menor dotación del recurso hídrico. Sin embargo, la comisión de Morrope es también aquella que posee una mayor cantidad de usuarios y predios bajo riego, por lo que la incidencia de factores externos, como robos y deficiencia en el control, es mayor y podría afectar los resultados obtenidos.

\section{Conclusiones}

Se ha constatado que la huella hídrica total de los cultivos estudiados en $\mathrm{m}^{3}$.ton ${ }^{-1}$ de producto generado se estima en 156 , 1124 y 891 para la cańa de azúcar, el arroz y el maíz respectivamente; con una contribución del componente verde en $1.6 \%, 0.7 \%$ y $3.4 \%$ para cada caso, siendo el resto de agua requerida a través del riego. El cultivo con mayor huella es el arroz, porque requiere de elevados volúmenes de agua para la preparación del suelo y para el riego por inundación, aunado a las pérdidas por evaporación y percolación profunda. La caña de azúcar es el cultivo con menor huella hídrica, explicado por altos de rendimiento en biomasa; en ese sentido, el consumo de agua debe evaluarse también respecto a la superficie cultivada y no solo en términos de volúmenes de producción. Si se analiza los cultivos con base en la cantidad de agua requerida por superficie cultivada, se aprecia que la cańa de azúcar es el cultivo que mayor consumo registra, al requerir $16000 \mathrm{~m}^{3} \cdot \mathrm{ha}^{-1}$, en tanto que el arroz y el maíz solo requieren 9000 y $6000 \mathrm{~m}^{3} \cdot \mathrm{ha}^{-1}$ de agua respectivamente.

En un escenario de precipitaciones nulas o con un porcentaje bajo de agua de lluvia incorporada, las mejoras deberían realizarse en función del mejor aprovechamiento de agua a través de los métodos de riego, sobre todo en el caso de la caña de azúcar y el arroz, porque el primero es el cultivo que más agua utiliza por hectárea, y el segundo es el cultivo más frecuente y con la menor eficiencia de riego. Se concluye, por tanto, que a la luz de los resultados que el cultivo más eficiente, económicamente, en cuanto al uso del agua es el arroz, al recaudar 5.10 soles por metro cúbico de agua consumida, mientras que la caña de azúcar posee el valor más bajo, generando solo 1.74 soles por metro cúbico. Por otro lado, el más eficiente respecto al uso de los suelos también es el arroz, porque recauda 10 380 soles por hectárea cultivada, siendo el maíz el menos eficiente al generar 6 159 soles por hectárea.

Estos resultados harían pensar que el arroz es el cultivo más rentable dentro de la región, pero es necesario recordar que los indicadores económicos utilizados como referencia de la ecoeficiencia solo consideran como impacto ambiental el consumo de agua, pero dejan de lado otro impactos relacionados, específicamente 
con el cultivo de arroz como su alto grado de contaminación por emisiones de $\mathrm{CH}_{4}$, o que está siendo cultivado en una región costera donde existen altos niveles de estrés hídrico y donde el agua posee un valor más importante que en otras zonas. Desde ese punto de vista, al no contempla un análisis más integral sobre la totalidad de impactos ambientales, intención última que escapa a los objetivos planteados en el presente estudio, pero permiten avizorar nuevas líneas de investigación en el ámbito de la valoración de la contaminación por medio de la ecoeficiencia.

\section{Referencias}

Abelardo de la Torre, Jefe de la Autoridad Nacional del Agua (2017) https:// agraria.pe/noticias/80-del-agua-enperu-se-destina-a-la-agriculturaurge-13448

Aldaya, M. (2015). Introducción al cálculo de la huella hídrica según Water Footprint Network: La huella bidrica como instrumento para la planificación hidrológica y reducción de conflictos. Seminario Nacional del Observatorio del Agua, Madrid.

Alexandratos, N., \& Bruinsma, J. (2012). World agriculture towards 2030/2050: the 2012 revision (Vol. 12 , No. 3). FAO, Rome: ESA Working paper.

Allan, J.A. (1998).Virtual water: a strategic resource. Ground water, 36(4), 545547.

Altieri, M. A., \& Nicholls, C. I. (2009). Cambio climático y agricultura campesina: impactos y respuestas adaptativas. LEISA revista de agroecología, 14, 5-8.

Álvarez, E., Boisán, M., Cabo, M., Chaves, E., \& Pozo, V. (2014). Los Túneles Pucará y Transandino del proyecto de trasvase Majes Siguas II, Arequipa, Perú. Ingeopres:
Actualidad técnica de ingeniería civil, minería, geología y medio ambiente, (233), 18-24.

Anuario Estadístico de Producción Agrícola (2017). Ministerio de Agricultura y Riego del Perú.

Arteaga, S.; Fierro, G. (2015). Valoración de la infraestructura de riego aplicado a Cuambo, Parroquia Salinas, Cantón Ibarra, Provincia de Imbabura.

Banco Mundial (2008). Informe sobre el desarrollo mundial 2008: Agricultura para el desarrollo (No. 338.9 B3Y 2008.). Washington, D.C. http:// econ.worldbank.org/wbsite/ external/extdec/extsearch

Bayart, J. B., Bulle, C., Deschênes, L., Margni, M., Pfister, S., Vince, F., \& Koehler, A. (2010). A framework for assessing off-stream freshwater use in LCA. The International Journal of Life Cycle Assessment, 15(5), 439-453.

Bedmar, F., Gianelli, V., Angelini, H., \& Vigilianchino, L. (2015). Riesgo de contaminación del agua subterránea con plaguicidas en la cuenca del arroyo El Cardalito, Argentina. RIA. Revista de investigaciones agropecuarias, 41(1), 70-82. 
Bedoya, V. H., \& López-Lezama, J. M. (2015). Modelo para el Control de Inundaciones durante el Fenómeno de" La Niña" Utilizando un embalse Hidroeléctrico. Información tecnológica, 26(2), 89-100.

Benavides, C., Ángel, V., Jara, V., \& Edgar, I. (2014). Comparación de tres sistemas de riego para la producción de arroz con tres densidades de siembra en Zamorano.

Benavides, M. (2014). Eficiencia técnicaeconómica y riesgo en la producción del cultivo de arroz.

Bolaños, M. E. (2011). Determinación de la huella hídrica y comercio de agua virtual de los principales productos agrícolas de Honduras. Zamorano, Honduras.

Bossio, D., Geheb, K., \& Critchley, W. (2010).Managingwaterbymanaging land: addressing land degradation to improve water productivity and rural livelihoods. Agricultural Water Management, 97(4), 536-542.

Boulay, A. M., Bare, J., Benini, L., Berger, M., Klemmayer, I., Lathuilliere, M., ... \& Ridoutt, B. (2014). Building consensus on a generic water scarcity indicator for LCA-based water footprint: preliminary results from WULCA. In Proceedings of the 9th International Conference on Life Cycle Assessment in the Agri-Food Sector (LCA Food 2014), San Francisco, California, USA, 8-10 October 2014 (pp. 142-149). American Center for Life Cycle Assessment.

Boulay, A. M., Bare, J., Benini, L., Berger, M., Lathuillière, M. J., Manzardo,
A., ... \& Ridoutt, B. (2018). The WULCA consensus characterization model for water scarcity footprints: assessing impacts of water consumption based on available water remaining (AWARE). The International Journal of Life Cycle Assessment, 23(2), 368-378.

Boulay, A. M., Motoshita, M., Pfister, S., Bulle, C., Muñoz, I., Franceschini, H., \& Margni, M. (2015). Analysis of water use impact assessment methods (part A): evaluation of modeling choices based on a quantitative comparison of scarcity and human health indicators. The international journal of life cycle assessment, 20 (1), 139-160.

Brundland, G. H. (1987). Informe de la comisión mundial sobre el medio ambiente y el desarrollo (Comisión Brundland): Nuestro futuro común. New York: Comisión Brundland.

Bunge, V. (2010). La presión hídrica en las cuencas de México. Las cuencas hidrográficas de México: diagnóstico y priorización. México: INE, 88-91.

Cadena, V. (2012). Hablemos de riego. Creadores gráficos. QuitoEcuador, 43.

Conesa Fernández, V., Ros Garro, V., Conesa Ripoll, V., \& Conesa Ripoll, L. A. (2010). Guia metodológica para la evaluación del impacto ambiental. Mundi-prensa.

De Fraiture, C., Molden, D., \& Wichelns, D. (2010). Investing in water for food, ecosystems, and livelihoods: 
An overview of the comprehensive assessment of water management in agriculture. Agricultural Water Management, 97(4), 495-501.

Effendi, H. (2016). River water quality preliminary rapid assessment using pollution index. Procedia Environmental Sciences, 33, 562-567.

Escobal, J., Revesz, B., \& Trivelli, C. (2006). Pequeña Agricultura Comercial: dinámica y retos en el Perú.

Fereres, E., Soriano, M.A., (2007).Deficit irrigation for reducing agricultural water use J. Exp. Bot. 58, 147-158

Ferrandis, P. (2006). El medio natural como receptor de impactos ambientales. La evaluación del impacto ambiental de proyectos $y$ actividades agroforestales, 632.

Food and Agriculture Organization of the United Nations. (2018). Principios para una agricultura sostenible. [online] Available at: http://www. fao.org/sustainability/background/ principles/es/

Franco, A V. (2018). Evaluación de la eficiencia del método de riego por goteo (Bachelor's thesis).

Frischknecht, R., Steiner, R., \& Jungbluth, N. (2009). The Ecological Scarcity Method-EcoFactors 2006-A method for impact assessment in LCA (Methode der ökologischen KnappheitÖkofaktoren 2006-Methode für die Wirkungsabschätzung in Ökobilanzen).
Geerts, S., \& Raes, D. (2009). Deficit irrigation as an on-farm strategy to maximize crop water productivity in dry areas. Agricultural water management, 96(9), 1275-1284.

Gleeson, T., Befus, K. M., Jasechko, S., Luijendijk, E., \& Cardenas, M. B. (2016). The global volume and distribution of modern groundwater. Nature Geoscience, 9(2), 161.

Hoekstra, A. Y., \& Chapagain, A. K. (2006). Water footprints of nations: water use by people as a function of their consumption pattern. In Integrated assessment of water resources and global change (pp. 3548). Springer, Dordrecht.

Hoekstra, A. Y., Chapagain, A. K., Aldaya, M. M., \& Mekonnen, M. M. (2011). The water footprint assessment manual. Setting the global standard, 1, 224.

Hoekstra, A. Y., Mekonnen, M. M., Chapagain, A. K., Mathews, R. E., \& Richter, B. D. (2012). Global monthly water scarcity: blue water footprints versus blue water availability. PLoS One, 7(2), e32688.

ISO 14045:2012 Environmental management - Eco-efficiency assessment of product systems - Principles, requirements and guidelines (2012)

ISO 14046:2014 Environmental management - Water footprint - Principles, requirements and guidelines (2014) 
Kounina, A., Margni, M., Bayart, J. B., Boulay, A. M., Berger, M., Bulle, C., ... \& Núñez, M. (2013). Review of methods addressing freshwater use in life cycle inventory and impact assessment. The International Journal of Life Cycle Assessment, 18(3), 707721.

Larrazabal, M (2019). Tipos de Agricultura ¿Cuáles Son y Cómo se Clasifican? https://www.bialarblog. $\mathrm{com} /$ tipos-de-agricultura-cualescomo-clasifican/

Larsen, M. C., Hamilton, P. A., \& Werkheiser, W. H. (2013). Water quality status and trends in the United States. Monitoring Water Quality, Elsevier, NC, EE. UU, 19-57.

Leal, J. (2005). Ecoeficiencia: marco de análisis, indicadores y experiencias. CEPAL.

MINAGRI (2015). Manual del Cálculo de la Eficiencia para Sistema de Riego. Dirección General de Infraestructura Agraria y Riego Ministerio de Agricultura y Riego del Perú.

Masip, J. C. (2001). La infraestructura hidráulica de regadío en Almería. In El sector agrario y agroalimentario de Almería ante el siglo XXI: evolución $y$ perspectiva de nuestra agricultura en el año 2000: producción integrada: incidencia de las nuevas normativas de residuos de plaguicidas sobre la horticultura almeriense (pp. 13-31). Instituto de Estudios Almerienses.

Martínez, A., Chargoy, J., Puerto, M., Suppen, N., Rojas, D., \& Alfaro,
S. (2016). Huella de Agua (ISO 14046) en América Latina, análisis y recomendaciones para una coherencia regional. Centro de Análisis de Ciclo de Vida y Diseño Sustentable CADIS, Embajada de Suiza en Colombia, Agencia Suiza para la Cooperación y el Desarrollo COSUDE, 90.

Massoud, M. A., Al-Abady, A., Jurdi, M., \& Nuwayhid, I. (2010). The challenges of sustainable access to safe drinking water in rural areas of developing countries: case of Zawtar El-Charkieh, Southern Lebanon. Journal of Environmental Health, 72(10).

McMichael, C. E. (2014). Freshwater. The Encyclopedia of Earth.

Motoshita, M., Ono, Y., Pfister, S., Boulay, A. M., Berger, M., Nansai, K. \& Inaba, A. (2018). Consistent characterisation factors at midpoint and endpoint relevant to agricultural water scarcity arising from freshwater consumption. The International Journal of Life Cycle Assessment, 23(12), 2276-2287.

Nieto, N. (2011). La gestión del agua: tensiones globales $\mathrm{y}$ latinoamericanas. Politica y cultura, (36), 157-176.

Pachauri, R. K., Allen, M. R., Barros, V. R., Broome, J., Cramer, W., Christ, R., ... \& Dubash, N. K. (2014). Climate change 2014: synthesis report. Contribution of Working Groups I, II and III to the fifth assessment report of the Intergovernmental Panel on Climate Change (p. 151). Ipcc. 
Pfister, S., \& Bayer, P. (2014). Monthly water stress: spatially and temporally explicit consumptive water footprint of global crop production. Journal of Cleaner Production, 73, 52-62.

Pfister, S., Boulay, A. M., Berger, M., Hadjikakou, M., Motoshita, M., Hess, T., ... \& Manzardo, A. (2017). Understanding the LCA and ISO water footprint: A response to Hoekstra (2016). "A critique on the water-scarcity weighted water footprint in LCA". Ecological Indicators, 72, 352-359.

Pfister, S., Koehler, A., \& Hellweg, S. (2009). Assessing the environmental impacts of freshwater consumption in LCA. Environmental science \& technology, 43(11), 4098-4104.

Ponce, V. M., Silva, J. A., \& Villarroya, J. P. (2018) ¿Cuánta agua puede ser bombeada de un acuífero? Congreso Latinoamericano de Hidráulica, Argentina.

Rees, W. E. (1992). Ecological footprints and appropriated carrying capacity: what urban economics leave out? Environment and urbanization, 4(2), 121-130.

Renderos, R. (2014) Huella Hidrica del Cultivo de Caña de Azúcar.

Rojas-Valencia, M. N., Gallardo-Bolaños, J. R., \& Martínez-Coto, A. (2012). Implementación y caracterización de un sistema de captación y aprovechamiento de agua de lluvia. TIP Revista Especializada en Ciencias Químico-Biológicas, 15(1), 16-23.
Salmoral, G., Dumont, A., Aldaya, M. M., Rodríguez-Casado, R., Garrido, A., \& Llamas, M. R. (2012). Análisis de la huella hidrica extendida de la cuenca del Guadalquivir. Fundación Marcelino Botín.

Subramani, A., \& Jacangelo, J. G. (2015). Emerging desalination technologies for water treatment: a critical review. Water research, 75, 164-187.

Teixeira, R. F., de Souza, D. M., Curran, M. P., Antón, A., Michelsen, O., \& i Canals, L. M. (2016). Towards consensus on land use impacts on biodiversity in LCA: UNEP/SETAC Life Cycle Initiative preliminary recommendations based on expert contributions. Journal of Cleaner Production, 112, 4283-4287.

Trivelli, C., Escobal \& J., Revesz, B. (2006). Pequeña Agricultura Comercial: dinámica y retos en el Perú.

UNESCO. Informe de las Naciones Unidas sobre el Desarrollo de los Recursos Hídricos en el Mundo, 2017. http://www.unesco.org/new/ es/natural-sciences/environment/ water/wwap/wwdr/

United Nations International Children's Emergency Fund. (2018). Agua, saneamiento e higiene. https:// www.unicef.org/spanish/wash/ index_31600.html/

Viñas, J. M. S. (2012). Los retos de la agricultura para alimentar al mundo en 2050. Tiempo de paz, 106, 37-48.

WEF, F. E. M. (2016). Global risks 2016. World Economic Forum. 
Williamson, O. E. (2002). The Theory of the Firm as Governance Structure: From Choice to Contract. Journal of Economic Perspectives 16 (3): 171-195.

World Health Organization (2006). Guidelines for the safe use of wastewater, excreta and greywater. Vol 1. Policy and regulatory aspects and Vol 2. Wastewater uses in agriculture.
WWF \& SABMiller. (2009). Water Footprinting: Identifing and addressing water risks in the value chain. Water Footprint Network.

Zhang, Y., \& Zhifeng, Y. (2007). Ecoefficiency of urban material metabolism: a case study. Acta Ecológica Sinica, 3124-3131. 